\title{
Оценка объема оттаявшей породы через интегрирование теплофизических параметров
}

\author{
Амосов П.В. \\ Институт проблем промышиенной экологии Севера КНЦ РАH, Anamumbl, p.amosov@ksc.ru
}

\begin{abstract}
Аннотация. Представлены результаты оценки объема оттаявшей породы при размещении в ней многомодульной атомной станции малой мощности через интегрирование плотности, объемной теплоемкости и теплопроводности по объему вмещающего массива и с учетом различий в значениях указанных теплофизических параметров для льда и воды. Для снижения погрешности оценки объема оттаявшей породы предложено выполнить операцию осреднения расчетных значений трех оценочных зависимостей. Получены аналитические зависимости в форме степенных функций динамики объема и скорости объема оттаявшей породы.

Ключевые слова: многомодульная атомная станция, теплофизические параметры, численное моделирование, интегрирование, криолитозона.
\end{abstract}

\section{Estimation of the volume of thawed rock by integration of thermophysical parameters}

\author{
Amosov P.V. \\ Institute of the Industrial Ecology Problems of the North KSC RAS, Apatity, p.amosov@ksc.ru
}

\begin{abstract}
The paper presents the results of estimating the volume of thawed rock when placing a small multimodule nuclear power plant in it by integrating density, volume heat capacity, and thermal conductivity over the volume of the host massif and taking into account the differences in the values of these thermophysical parameters for ice and water. To reduce the error in estimating the volume of thawed rock, it is proposed to perform the operation of averaging the calculated values of the three estimated dependencies. Analytical dependences in the form of power functions of the volume dynamics and the volume velocity of the thawed rock are obtained.

Key words: multi-module nuclear power plant, thermophysical parameters, numerical modeling, integration,
\end{abstract} cryolithozone.

\section{Введение}

Настоящая работа завершает цикл исследований автора с коллегами (Мельников и др., 2016; Амосов, 2019) по оценке объема оттаявшей породы при размещении в многолетнемерзлых породах разнообразных источников тепловыделений. Такими объектами могут быть хранилища хранения/захоронения опасных тепловыделяющих материалов, подземная атомная станция малой мощности (ACMM) и др. Указанные исследования в отношении АСММ выполнялись в рамках оценки теплового воздействия подземной АСММ на многолетнемерзлые горные породы (Мельников и др., 2015). Оценка теплового воздействия являлась отдельной задачей в проблеме изучения взаимодействия подземной АСММ в режиме нормальной эксплуатации с окружающей средой. Цель прогнозов теплового состояния вмещающего массива направлена на поиск условий и технологических мероприятий, обеспечивающих устойчивость и целостность создаваемых в криолитозоне подземных горных выработок.

Попытки автора оценить объемы оттаивания исходя исключительно из геометрии изоповерхности с температурой фазового перехода «лед - вода» были неудачными, как только усложнялась конфигурация подземной АСММ. Поэтому автор с коллегами предложили и апробировали методический подход (Мельников и др., 2016; Амосов, 2019), базирующийся на операции интегрирования плотности, объемной теплоемкости и теплопроводности по объему многолетнемерзлых пород. Важным моментом здесь является то, что в значениях указанных параметров воды и льда имеются заметные различия. Предложив разместить все модули подземной АСММ в одной горной выработке (камера большого объема), удалось через интегрирование указанных выше параметров оценить (в форме аналитических функций) объем и скорость объема оттаивания многолетнемерзлых пород. 
Параметрами варьирования выступали продольный размер выработки и коэффициент теплопроводности обделки. Заметим, что принцип размещения в едином корпусе всех модулей станции используется в проекте плавучей атомной теплоэлектростанции «Академик Ломоносов» (Общие сведения ..., 2014; Никитин, Андреев, 2011).

В предыдущих работах автора с коллегами аккуратно расписаны алгоритмы и выполнены оценки объема области оттаивания через интегрирование плотности (Мельников и др., 2016) и объемной теплоемкости (Амосов, 2019) по объему криолитозоны. Как отмечалось выше, помимо указанных теплофизических параметров есть еще один - коэффициент теплопроводности, значения которого для льда и воды имеют величины 2.31 и $0.613 \mathrm{BT} /($ мКК), соответственно.

В отличие от указанных выше и реализованных ранее подходов в случае интегрирования по объему коэффициента теплопроводности приходится оперировать величиной, имеющей нетривиальную размерность, а именно [Вт $\left.\mathrm{M}^{2} / \mathrm{K}\right]$. Будем называть эту величину условной интегральной теплопроводностью и обозначать символом $\Lambda$. В отличие от интегрирования плотности (результат - масса [кг]) или объемной теплоемкости (результат - условная энтропия [Дж/К]) в данном случае автор затрудняется найти аналог или физический смысл этого параметра, но для конечного результата это непринципиально.

В 2015 г. сотрудники Горного института Кольского научного центра РАН (Мельников, 2015) предложили принципиальную схему многомодульной подземной АСММ. Компоновка модулей станции, которая включала помещения для реакторных установок, машинного зала, технических служб и др. представлена на рис. 1. Именно для такого варианта компоновки подземной АСММ выполнены оценки объема оттаявшей породы через интегрирование всех указанных выше теплофизических параметров по объему вмещающего массива и представлен сравнительный анализ результатов.

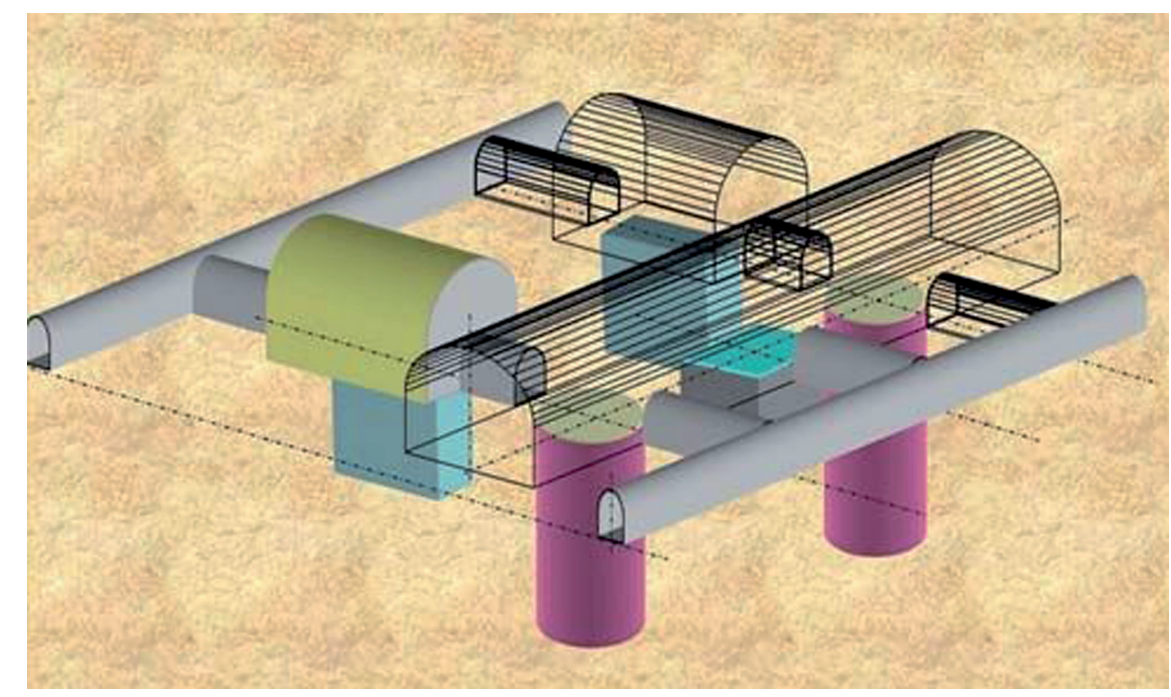

Рис. 1. Принципиальная схема подземной атомной станции малой мощности (Мельников, 2015, с. 35).

Fig. 1. Schematic diagram of a small underground nuclear power plant (Melnikov, 2015, p. 35).

Целью исследования является качественная и количественная оценка объема оттаявшей многолетнемерзлой горной породы во времени при функционировании многомодульной подземной АСММ в режиме нормальной эксплуатации через интегрирование всех трех теплофизических параметров. Исследования выполнялись на базе разработанной компьютерной объемной модели процессов теплопереноса в криолитозоне с учетом фазового перехода 1-го рода «лед - вода».

\section{Описание компьютерной модели и алгоритма подхода через интегрирование коэффициента теплопроводности}

Наиболее полно описание используемой компьютерной модели, разработанной в программе COMSOL (COMSOL, 2014), представлено в работах (Амосов, 2019; Мельников и др., 2020). Среди 
модулей подземной АСММ приняты во внимание наиболее крупные и важные модули. Напомним, что, как и в разработанной Казаковым А.Н. с коллегами программе (Казаков и др., 1997), используемый программный продукт построен на корректных объективных допущениях, не противоречащих классикам вычислительной теплопередачи (Самарский, Вабищевич, 2003).

Для сравнительного анализа результатов интегрирования всех трех теплофизических параметров выбрана ситуация максимальной презентабельности графических результатов, что обеспечивается выбором высокого значения коэффициента теплопроводности обделки (2 Вт/(м.К)) и минимальной величины пористости вмещающего массива (5 \%). В коллективной монографии (Мельников и др., 2020) на рис. 3.11a (стр.117) представлены пространственные распределения изоповерхностей температуры на 5 лет моделирования для различных значений коэффициента теплопроводности обделки. Подобные изображения позволяют визуализировать пространственные проявления областей теплового воздействия на массив от соседних модулей АСММ, но в силу сложной геометрии зон оттаивания практически невозможно оценить их объемы, опираясь только на их геометрические характеристики.

В работах (Мельников и др., 2016) и (Амосов, 2019) приведены аккуратные выводы аналитических выражений, посредством которых можно оценить объемы оттаивания криолитозоны через интегрирование плотности и объемной теплоемкости, соответственно. Алгоритм оценки объема оттаивания через интегрирование по криолитозоне коэффициента теплопроводности во многом повторяет описанные в указанных работах выкладки. Поэтому представим только окончательную формулу, которая справедлива на произвольный момент времени,

$$
\cdot \Delta \Lambda=V_{w} \cdot\left(\lambda_{w}-\lambda_{i}\right)
$$

В приведенном выражении в левой части имеем изменение условной интегральной теплопроводности (см. выше). В правой части используются стандартные обозначения: $V_{w}-$ объем воды в оттаявшем поровой пространстве, $\lambda_{w}-$ коэффициент теплопроводности воды и $\lambda_{i}-$ коэффициент теплопроводности льда.

Очевидно, что если теперь разделить изменение условной интегральной теплопроводности на разность коэффициентов теплопроводности воды и льда, то получим величину объема оттаивания на произвольный момент времени. Заметим, что в отличие от подходов интегрирования плотности и объемной теплоемкости (обе величины определяются на единицу объема) в данном случае имеем объем воды. А значит, чтобы выйти на объем оттаявшей породы, требуется поправить рассчитанную величину на пористость вмещающего массива.

\section{Результаты анализа оценки объема оттаявшей породы}

Разработчики COMSOL (COMSOL, 2014) заложили в программу полезную опцию, позволяющую выполнять операцию интегрирования по объему различных областей модели (в т.ч. и криолитозоне) указанных выше теплофизических параметров. При изменении во времени объема оттаявшей породы интегральные величины будут изменяться. Анализ динамики интегральных величин (масса, условная интегральная энтропия, условная интегральная теплопроводность) показал, что все они с коэффициентами достоверности близкими к единице описываются степенными функциями вида $V=A \cdot t^{B}$, где $V$ - объем оттаявшей породы, ${ }^{3} ; t$ - время, годы. Значения коэффициентов аппроксимации $A$ и $B$ для используемых теплофизических параметров приведены в таблице.

На рис. 2 представлены графики динамики аппроксимации объема оттаявшей породы при интегрировании плотности, теплоемкости и теплопроводности по объему вмещающего массива.

Действительно, имеет место определенное расхождение графиков, что позволяет утверждать, что такой подход можно рассматривать лишь как оценку. Представляется, что расхождение графиков может быть связано с неточностями вычислений при выполнении операции интегрирования по объему вмещающего массива. С целью снижения ошибки оценки выполнено осреднение приведенных трех графиков. В результате имеем график Medium, который можно рассматривать, как осредненную оценку объема оттаявшей породы и который описывается такой же степенной функцией с коэффициентами $A=225215$ и $B=0.5719$. 


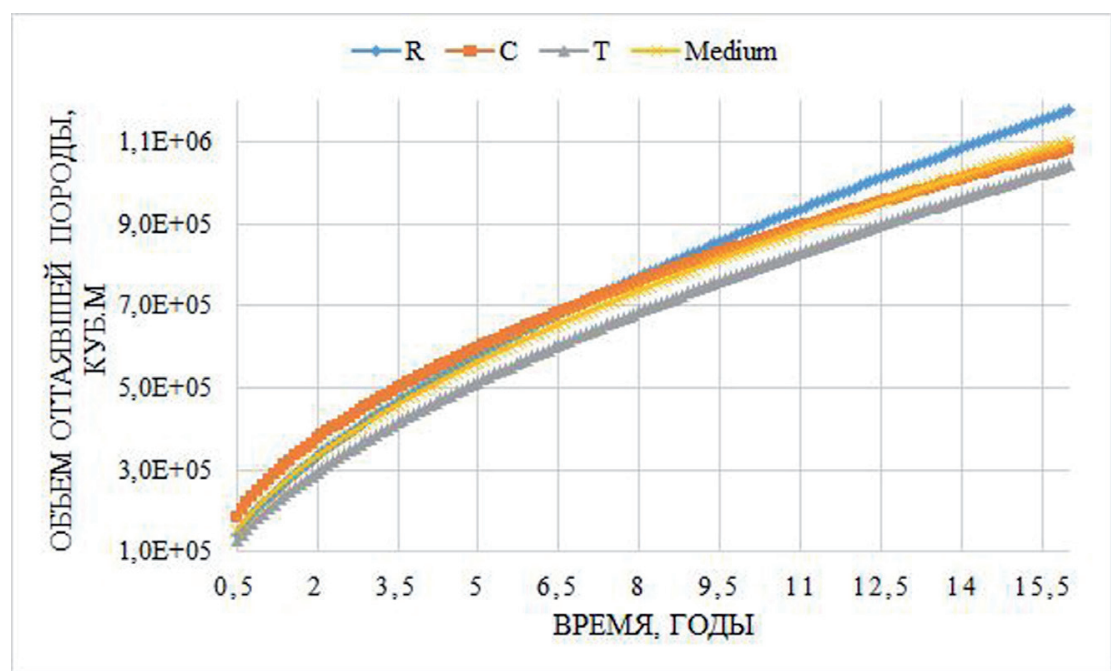

Рис. 2. Кривые динамики аппроксимации объема оттаявшей породы при интегрировании плотности, теплоемкости и теплопроводности по объему вмещающего массива: $\mathrm{R}$ - плотность, $\mathrm{C}$ - теплоемкость, Т - теплопроводность, Medium - среднее значение.

Fig. 2. Curves of approximation dynamics for the volume of thawed rock by integrating the density, heat capacity, and thermal conductivity over the volume of the host massif: $\mathrm{R}$ - the density, $\mathrm{C}$ - heat capacity, $\mathrm{T}$ - the thermal conductivity, Medium - the mean value.

Таблица. Значения коэффициентов $A$ и $B$ в степенных функциях аппроксимации.

Table. The values of coefficients $A$ and $B$ in the power approximation functions.

\begin{tabular}{|c|c|c|c|}
\hline \multirow{2}{*}{ Коэффициенты } & \multicolumn{3}{|c|}{ Теплофизические параметры } \\
\cline { 2 - 4 } & плотность & теплоемкость & теплопроводность \\
\hline$A$ & 218945 & 263024 & 194550 \\
\hline$B$ & 0.6065 & 0.5098 & 0.6063 \\
\hline
\end{tabular}

Естественно, что имея на руках аналитическое выражение осредненной динамики объема оттаявшей породы, легко получаем аналитическую зависимость для скорости объема оттаивания $V S=128801 \cdot t^{-0.4281}, \mathrm{M}^{3} /$ год. Полученное выражение позволяет оценить скорость объема оттаивания на произвольное время, которая с ростом времени постепенно замедляется, что физически верно.

\section{Заключение}

Таким образом, на примере многомодульной подземной АСММ в многолетнемерзлых горных породах представлены результаты оценки объема и скорости объема оттаявшей породы посредством интегрирования плотности, объемной теплоемкости и теплопроводности по объему вмещающего массива. Результаты оценки, основанные на природных различиях в значениях указанных теплофизических параметров для льда и воды, имеют определенный разброс значений, что может быть нивелировано последующим осреднением. Для принятых в численных экспериментах значениях параметров модели получены в форме степенных функций аналитические зависимости динамики объема и скорости объема оттаявшей породы.

\section{Литература}

1. Амосов П.В. Оценка размера области оттаивания криолитозоны через интегрирование объемной теплоемкости по объему вмещающего массива // Горный информационно-аналитический бюллетень. № 11. Спец.вып. 37. 2019. С. 457-465. DOI:10.25018/0236-1493-2019-11-457-465.

2. Казаков А.Н., Лобанов Н.Ф., Манькин В.И. Динамика развития теплофизических процессов при подземной изоляции тепловыделяющих РАО в многолетнемерзлых горных породах // Геоэкология. 1997. № 2. C. $36-40$. 
3. Мельников Н.Н. Роль Арктики в инновационном развитии экономики России // Горный журн. 2015. № 7. C. 23-27. http://dx.doi.org/10.17580/gzh.2015.07.04.

4. Мельников Н.Н., Амосов П.В., Климин С.Г., Новожилова Н.В. Оценка области оттаивания криолитозоны при эксплуатации подземной АСММ на базе численного моделирования в трехмерной геометрии // Вестник МГТУ. 2016. Т. 19. № 1-1. С. 28-34.

5. Мельников Н.Н., Амосов П.В., Новожилова Н.В., Климин С.Г. Экология подземных объектов ядерной энергетики в условиях криолитозоны. Ярославль. Изд-во: ООО «Принтхаус-Ярославль». 2015. 119 с.

6. Мельников Н.Н., Конухин В.П., Гусак С.А., Амосов П.В., Наумов В.А., Наумов А.В., Орлов А.О., Смирнов Ю.Г., Климин С.Г. Научные основы создания подземных комплексов для размещения атомных станций малой мощности в условиях Арктики. Апатиты. Изд-во: КНЦ РАН. 2020. 304 с. DOI:10.37614/978.5.91137.405.1.

7. Никитин А., Андреев Л. Плавучие атомные станции. Доклад объединения Bellona. 2011. 48 с.

8. Общие сведения о проекте энергокомплекса на базе плавучего энергоблока с реакторными установками КЛТ-40С сайт с ПАТЭС. URL: http://shkolnie.ru/fizika/39130/index.html (дата обращения 22.06 2014).

9. Самарский А.А., Вабищевич П.Н. Вычислительная теплопередача. М. Изд-во: Едиториал УРСС. 2003. $784 \mathrm{c}$.

10. COMSOL. URL:https://www.comsol.ru/ (дата обращения 12.01 2014). 